\title{
Successful Use of Sugammadex in a Myasthenic Patient Case Report
}

\author{
Silvia Kangassu Rios, Daiana Gomes, Marcos Lopes De Miranda, Carlos Frederico La Cava, \\ Carlos Darcy Bersot
}

Department of Anesthesia, Hospital Federal da Lagoa, Rio de Janeiro, Brazil.

Email: carlosbersot@gmail.com

Received October $16^{\text {th }}, 2012$; revised November $20^{\text {th }}, 2012$; accepted December $30^{\text {th }}, 2012$

\begin{abstract}
Myasthenia gravis (MG) is an autoimmune disease that affects neuromuscular transmission and leads to a large variation of sensibility on depolarizing and nondepolarizing neuromuscular blocking drugs. Sugammadex is a new drug with the capability to successfully revert the nondepolarizing neuromuscular blocking. This case report will show a patient with MG that was scheduled for thymectomy, with the objective to use sugammadex to reverse a rocuronium induced deep level of neuromuscular block and observing the safe use of sugammadex without complications.
\end{abstract}

Keywords: Sugammadex; Myasthenia Gravis; Rocuronium; Neuromuscular Monitoring; Anesthesia.

\section{Introduction}

Myasthenia gravis is an autoimmune disease caused by the blockade of neuromuscular transmission by antibodies against nicotinic acetylcholine receptors (AChRs), which are located in the postsynaptic membrane of the neuromuscular junction. Because of the small number of normal AChRs, MG patients present an abnormal reaction to neuromuscular depolarizing blocking agents, and this patients can have prolonged postoperative muscle relaxation, leading to risk of respiratory failure in post operative period. MG occurs in about 20 people per 100,000 usually only sporadically [1].

Major clinical importance is the classification of myasthenia gravis developed by Osserman and Genkins [2]. In 1958, the division of patients with myasthenia gravis in five groups: 1) localized (ocular); 2) generalized (mild or moderate); 3) acute fulminating; 4) late severe; and 5) muscle atrophy. Later, Osserman and Genkins divided group 2) into the following subclassifications: A (mild) and B (moderate). This classification allows one to grade the disease and assess the perioperative risk and possible complications.

Sugammadex is a modificated g-cyclodextrin (refers to sugar and gammadex refers to the structural molecule gcyclodextrin). It is a selective relaxant binding agent. It binds/encapsulates and renders inactive the steroidal neuromuscular blocking agents (NMBA) rocuronium, and vecuronium.

This decreases the concentration of unbound NMBA at the neuromuscular junction thereby reversing NMB [3].
Sugammadex has been administered and showed safe for use in patients with myasthenia gravis and may effectively reduce the risk of postoperative respiratory complications, but further research is needed to clarify key parameters in the analysis and to allow a fuller economic assessment.

\section{Case Report}

A 21-year-old female patient (weight $50 \mathrm{~kg}$, height 156 $\mathrm{cm}$ ), who stated no allergies. The patient has been using levonorgestrel $0.15 \mathrm{mg}$ and etinilestradiol $0.03 \mathrm{mg}$ since 2005. In 2008 the patient developed progressive bilateral ptosis and eye deviation with diplopia without other associated symptoms.

In 2009 the patient underwent detection of acetylcholine receptor antibodies that were $7.4 \mathrm{nmol} / \mathrm{l}$. The severity of myasthenia was determined as class I (ocular myasthenia) as per Osserman and Genkins's classification.

At the time of diagnosis the patient was treated with prednisone and pyridostigmine for 8 months. After, the patient was treated with pyridostigmine $360 \mathrm{mg}$ and azathioprine $50 \mathrm{mg}$ day for 1 year and 2 months. The azathioprine was suspended 2 months before the thymectomy and the pyridostigmine was suspended 6 hours before the surgery. The patient was in optimal condition for surgery and without any other abnormalities. TC scan was compatible with thymic-hyperplasia.

After the patient consent was obtained, the patient was monitored with pulse oximetry, electrocardiogram, noninvasive arterial blood pressure, capnography, Bispectral 
index, body temperature (nasopharyngeal). The neuromuscular function was monitored at the adductor pollicis muscle using train-of-four (TOF) with acceleromyography.

Following pre-oxygenation the patient was induced with $150 \mathrm{mcg}$ fentanyl and $100 \mathrm{mg}$ propofol. $15 \mathrm{mg}$ rocuronium was administered to achieve a maximum block of T1 for a TOF stimulus. The intubation conditions were excellent.

In our case, rocuronium was used to provide optimal conditions for intubation. After infusion of rocuronium, heart rate, TOF, BIS and non-invasive pressure were measured, as shown in Table 1.

Ten minutes after intubation, it was used sugammadex $8 \mathrm{mg} / \mathrm{kg}$, until the reversal of NMB, the following parameters were analyzed in intervals of 20 seconds: TOF (train on four), BIS (bispectral index), heart rate and invasive blood pressure. It was observed an increasing in the BIS and minimum hemodynamic consequences, as one can observes in Table 2.

In the 150 minutes of surgery, the TOF remained above 90\%. Epidural anesthesia was accomplished in T9 $\mathrm{T} 10$ and ropivacaine $0.5 \%$ - $10 \% \mathrm{mg}$ was administered.

The patient was transferred to the intensive care unit where clinically neuromuscular function was monitored every hour. After 24 hours she was discharged to the ward and left hospital 7 days after the operation.

\section{Discussion}

Myasthenia gravis is a potentially serious but treatable organ specific autoimmune disorder characterized by weakness and fatigability of the voluntary muscles that is caused by autoantibodies against the nicotinic acetylcholine receptor (AChR) on the postsynaptic membrane at the neuromuscular junction.

The major abnormalities of the neuromuscular junction in myasthenia gravis include 1) reduced number of the AChRs leading to reduced length of the postsynaptic membrane; 2) shortening of the synaptic folds due to destruction of the terminal expansions; and 3) widening

Table 1. Parameters during anesthesia.

\begin{tabular}{ccccc}
\hline $\begin{array}{c}\text { Parameters after } \\
\text { rocuronium infusion }\end{array}$ & $\begin{array}{c}\text { Heart } \\
\text { rate }\end{array}$ & TOF & BIS & $\begin{array}{c}\text { Blood } \\
\text { pressure }\end{array}$ \\
\hline 1 minute after & 65 & 80 & 47 & $100 \times 70$ \\
2 minutes after & 67 & 60 & 42 & $90 \times 60$ \\
3 minutes after & 69 & 39 & 42 & $80 \times 60$ \\
4 minutes after & 72 & 27 & 40 & $80 \times 58$ \\
5 minutes after & 68 & 23 & 33 & $80 \times 60$ \\
6 minutes after & 68 & 18 & 35 & $80 \times 60$ \\
\hline
\end{tabular}

Table 2. Parameters after sugammadex infusion.

\begin{tabular}{ccccc}
\hline & $\begin{array}{c}\text { Heart } \\
\text { rate }\end{array}$ & TOF & BIS & $\begin{array}{c}\text { Blood } \\
\text { pressure }\end{array}$ \\
\hline At the moment infusion & 70 & 20 & 41 & $90 \times 70$ \\
20 seconds after & 82 & 32 & 57 & $90 \times 60$ \\
40 seconds after & 95 & 57 & 56 & $98 \times 76$ \\
60 seconds after & 109 & 84 & 64 & $92 \times 70$ \\
80 seconds after & 98 & 91 & 70 & $94 \times 70$ \\
100 seconds after & 88 & 95 & 75 & $90 \times 70$ \\
\hline
\end{tabular}

of the synaptic clefts caused by the shortening of the junctional folds. These changes are brought about by autoimmune attack on the postsynaptic membrane.

Antibodies directed against the post-synaptic neuromuscular junction protein, muscle specific kinase (MuSK) are found in a small proportion of generalized myasthenia gravis (MuSK-MG) patients. MuSK is a receptor tyrosine kinase which is essential for clustering of the acetylcholine receptors (AChRs) at the neuromuscular junction, but the mechanisms by which MuSK antibodies (MuSK-Abs) affect neuromuscular transmission are not clear. Overall, these results suggest that MuSK antibodies act in at least two ways. Firstly by indirectly affecting MuSK's ability to maintain the high density of AChRs and secondly by interfering with a compensatory presynaptic mechanism that regulates quantal release and helps to preserve neuromuscular function. These results raise questions about how MuSK is involved in retrograde signaling, and the combination of post-synaptic defects with lack of presynaptic compensation may begin to explain the more severe disease in MuSK-MG patients.

Myasthenia gravis patients, particularly those undergoing major surgery require special individual management in preparation for surgery, appropriate selection and administration of anaesthesia, and close postoperative monitoring, because this patients can have prolonged postoperative muscle relaxation, leading to risk of respiratory failure in the postoperative period.

Surgery and anaesthesia in myasthenic patients are associated with an increased risk of death and severe complications [4]. So one should observe every details.

It is known that the use of neuromuscular blocking agents for tracheal intubation diminishes the incidence of adverse postoperative upper airway symptoms, results in better tracheal intubation conditions, and reduces the rate of adverse haemodynamic events [5]. In myasthenic patient this becomes even more important.

Regional analgesia may reduce or eliminate the need for muscle relaxants in thoracic surgery. Epidural analgesia also offers the advantage of postoperative pain 
control with minimal or no opioid use. Epidural anesthesia offers a better postoperative pain control and respiratory function, and can minimize the need for NMBA during surgery.

There is a lack of knowledge of correct dosage of sugammadex, many studies have demonstrated a dose-response relationship with sugammadex for reversal of neuromuscular blockade in patients the use of $2 \mathrm{mg} / \mathrm{kg}, 4$ $\mathrm{mg} / \mathrm{kg}, 8 \mathrm{mg} / \mathrm{kg}$ and $16 \mathrm{mg} / \mathrm{kg}$ [6]. In our case we used 8 $\mathrm{mg} / \mathrm{kg}$.

In a Cochrane review Abrishami et al. [7], reported that sugammadex provides a more rapid reversal of rocuronium induced NMB than that provided by neostigmine independent of depth of blockade. In addition, there was no evidence of a diference in the number of reported adverse events found between sugammadex, neostigmine and placebo.

Furthermore, it is probable that the use of sugammadex in combination with appropriate neuromuscular monitoring and correct dosing will significantly reduce the occurrence of postoperative residual curarization (PORC), the incidence of PORC ranges from $5 \%$ to $40 \%$ despite the use of neostigmine and objective neuromuscular monitoring.

Prescribers need to be aware that sugammadex may decrease progestogen concentrations, similar to the decrease observed after missing a daily dose of an oral contraceptive. Women on the pill should refer to the missed dose advice for their contraceptive. Likewise, women using non-oral hormonal contraceptives, such as depot formulations, should be advised to use additional contraception for the next seven days. Our patient was aware.

Some early studies reported rare cases of prolongation of QTc interval when sugammadex was used alongside sevoflurane or propofol however 2 studies on non-anaesthetised healthy volunteers demonstrated no increase in QTc interval with dose of sugammadex with and without rocuronium or vecuronium. Two incidents of possible bleeding complications have been investigated by the European Medicines Agency. However a recent retrospective study found no increased postoperative bleeding when sugammadex was used.

In clinical practice, have been noticed a rise in the numerical values of bispectral index (BIS) and Entropy during the reversal of the NMB with sugammadex. In some patients, administration of sugammadex or neostigmine caused a signicant rise in the numerical values of BIS and Entropy. This phenomenon is most likely caused by increased electromyographic (EMG) activity. The administration of sugammadex or neostigmine ap- peared to have only minimal effect on EEG [8].

\section{Conclusions}

We successfully used sugammadex as an antagonist of muscle relaxants in patients with myasthenia gravis. This suggests that sugammadex use immediately after the intubation, in surgery that requires no relaxation, can prevent complications such as postoperative respiratory failure.

Furthermore, sugammadex in combination with appropriate neuromuscular monitoring showed no occurence of postoperative residual curarization.

\section{REFERENCES}

[1] L. H. Phillips II, “The Epidemiology of Myasthenia Gravis," Annals of the New York Academy of Sciences, Vol. 998, No. 1, 2006, pp. 407-412.

[2] K. Osserman and G. Genkins, "Studies in Myasthenia Gravis-Review of a 20-Year Eperience in over $1200 \mathrm{~Pa}-$ tients,” Mount Sinai Journal of Medicine, Vol. 38, No. 6, 1971, pp. 497-537.

[3] S. J. A. Gold and N. J. N. Harper, "The Place of Sugammadex in Anaesthesia Practice," Trends in Anaesthesia and Critical Care, Vol. 2, No. 1, 2012, pp. 4-9.

[4] F. X. Dillon, "Anesthesia Issues in the Perioperative Management of Myasthenia Gravis," Seminars in Neurology, Vol. 24, No. 1, 2004, pp. 83-94. doi:10.1055/s-2004-829587

[5] X. Combes, L. Andriamidy, E. Dufresne, P. Suen, S. Sauvat, E. Scherrer, P. Feiss, J. Marty and P. Duvaldestin, "Comparison of Two Induction Regimens Using or Not Using Muscle Relaxant: Impact on Postoperative Upper Airway Discomfort," British Journal of Anaesthesia, Vol. 99, No. 2, 2007, pp. 276-281. doi:10.1093/bja/aem147

[6] P. Duvaldestin, K. Kuizenga, V. Saldien, C. Claudius, F. Servin, J. Klein, B. Debaene and M. Heeringa, "A Randomized, Dose-Response Study of Sugam-Madex Given for the Reversal of Deep Rocuronium- or VecuroniumInduced Neuromuscular Blockade under Sevourane Anesthesia,” Anesthesia and Analgesia, Vol. 110, No. 1, 2010, pp. 74-82. doi:10.1213/ANE.0b013e3181c3be3c

[7] A. Abrishami, J. Ho, J. Wong, L. Yin and F. Chung, Sugammadex, "A Selective Reversal Medication for Preventing Postoperative Residual Neuromuscular Blockade,” Anasthesia \& Analgesia, Vol. 110, 2010, p. 1239.

[8] A. J. Aho, K. Kamata, A. Yli-Hankala, L. P. Lyytikinen, A. Kulkas and V. Jntti, "Elevated Bis and Entropy Values after Sugammadex or Neostigmine: An Electroencephalographic or Electromyographic Phenomenon?” Acta Anaesthesiologica Scandinavica, Vol. 56, No. 4, 2012, pp. 465-473. doi:10.1111/j.1399-6576.2011.02647.x 